\title{
Systems of linear forms and covers for star bodies
}

by

\author{
M. M. Dodson and S. Hasan (York)
}

In this paper Diophantine approximation involving general distance and error functions will be considered for systems of linear forms. Distance functions will be denoted by $F: \mathbb{R}^{n} \rightarrow \mathbb{R}$ and are continuous, non-negative and satisfy

$$
F(u \boldsymbol{x})=u F(\boldsymbol{x})
$$

for all $u \geq 0$ ([3], p. 103). An error function $\psi: \mathbb{R}^{n} \backslash\{\mathbf{0}\} \rightarrow \mathbb{R}$ is positive and satisfies $\psi(\boldsymbol{x}) \rightarrow 0$ as $|\boldsymbol{x}| \rightarrow \infty$, where $|\boldsymbol{x}|=\max \left\{\left|x_{1}\right|, \ldots,\left|x_{n}\right|\right\}$. An upper bound depending on a cover for a star body associated with the distance function $F$ (see [8]) and a restriction $\psi_{1}$ of the error function $\psi$ for the Hausdorff dimension of sets of linear forms (regarded as points in $\mathbb{R}^{m n}$ ) is obtained. The Hausdorff dimension is also expressed in terms of the Hausdorff dimension of sets of vectors (in $\mathbb{R}^{n}$ ) and of the lower order (defined below) of the error function $\psi_{1}$ (corresponding to the case $m=1$ ) when the functions $\psi$ and $\psi_{1}$ satisfy suitable decay conditions. This combines and extends the results of [4], [5] and [13]. Definitions and properties of Hausdorff dimension are given in [7].

First some notation and definitions are needed. Throughout $m, n$ and $N$ will be positive integers and $\boldsymbol{q} \in \mathbb{Z}^{m}$. The system

$$
a_{1} x_{1 j}+\ldots+a_{m} x_{m j}, \quad 1 \leq j \leq n,
$$

of $n$ real linear forms in $m$ real variables $a_{1}, \ldots, a_{m}$ will be written more concisely as $\boldsymbol{a} X$ where $\boldsymbol{a} \in \mathbb{R}^{m}$ and where $X$ is an $m \times n$ real matrix. The set of real $m \times n$ matrices will be identified with $\mathbb{R}^{m n}$ in the usual way (i.e., by identifying the matrix $X=\left(x_{i j}\right)$ with the point $\left(x_{11}, \ldots, x_{1 n}, x_{21}, \ldots, x_{m n}\right)$ in $\left.\mathbb{R}^{m n}\right)$. For each $\boldsymbol{x}$ in $\mathbb{R}^{n}$, denote the symmetrised fractional part of $\boldsymbol{x}$ by $\langle\boldsymbol{x}\rangle=\boldsymbol{x}-\boldsymbol{k}$, where $\boldsymbol{k}$ is the unique integer vector such that $\boldsymbol{x}-\boldsymbol{k} \in$ $\left(-\frac{1}{2}, \frac{1}{2}\right]^{n}$. Thus

$$
|\langle\boldsymbol{x}\rangle|=\max \left\{\left\|x_{1}\right\|, \ldots,\left\|x_{n}\right\|\right\},
$$


where for each real $u,\|u\|=\min \{|u-k|: k \in \mathbb{Z}\}$. Given an error function $\psi: \mathbb{R}^{n} \backslash\{\mathbf{0}\} \rightarrow \mathbb{R}^{+}$, define $\psi_{1}: \mathbb{R} \backslash\{0\} \rightarrow \mathbb{R}^{+}$by

$$
\psi_{1}(u)=\psi(u, 0, \ldots, 0) .
$$

Thus the function $\psi_{1}$ is essentially the restriction of $\psi$ to $(\mathbb{R} \backslash\{0\}) \times\{0\} \times$ $\ldots \times\{0\}$, so that $\psi_{1}$ is positive and $\psi_{1}(x) \rightarrow 0$ as $x \rightarrow \infty$. Functions $\Delta:[0, \infty) \rightarrow(0, \infty)$, where $\Delta(0)=1$ and $\Delta(x) \rightarrow \infty$ monotonically as $x \rightarrow \infty$, which satisfy the growth conditions

$$
\frac{\log \Delta(x)}{x} \rightarrow 0 \text { as } x \rightarrow \infty \text { and } \int_{1}^{\infty} \frac{\log \Delta(x)}{x^{2}} d x<\infty,
$$

characterise a large class of functions arising in small denominator problems and are called approximation functions ([11], p. 95). In the applications considered below, $\psi_{1}(x)=x^{-\tau}$; its reciprocal $1 / \psi_{1}$ is essentially an approximation function in the sense of [11] (strictly speaking $(x+1)^{\tau}$ is an approximation function). The lower order $\lambda=\lambda(f)$ of the function $f: \mathbb{R}^{+} \rightarrow \mathbb{R}^{+}$ is defined by

$$
\lambda=\liminf _{x \rightarrow \infty} \frac{\log f(x)}{\log x}
$$

([9], p. 16), so that given $\varepsilon>0, f(x) \geq x^{\lambda-\varepsilon}$ for all sufficiently large $x$.

Suppose that for each positive $r, \mathcal{C}_{r}$ is a collection of hypercubes $C$ in $\mathbb{R}^{n}$ with $\ell(C) \ll R$ (as usual $\ll$ denotes an inequality with an unspecified positive constant factor). The $s$-volume $\ell^{s}(\mathcal{C})$, where $s \geq 0$, of a collection $\mathcal{C}$ of hypercubes $C$ is given by

$$
\ell^{s}(\mathcal{C})=\sum_{C \in \mathcal{C}} \ell(C)^{s}
$$

(see [7]). For each $s$ in $[0, n]$, the (upper) order $\omega(s)$ of the $s$-volume $\ell^{s}\left(\mathcal{C}_{r}\right)$ of $\mathcal{C}_{r}$ will be defined as

$$
\omega(s)=\liminf _{r \rightarrow 0+} \frac{\log \ell^{s}\left(\mathcal{C}_{r}\right)}{\log r},
$$

so that given $\varepsilon>0, \ell^{s}\left(\mathcal{C}_{r}\right) \leq r^{\omega(s)-\varepsilon}$ for all sufficiently small $r$. It is shown in Lemma 1 of [5] that $\omega(s)$ increases with $s$ and is concave (i.e., $\omega$ satisfies $\omega(t a+(1-t) b) \geq t \omega(a)+(1-t) \omega(b)$ for each $a, b$ in $(0, n)$ and $t$ in $[0,1])$ and hence continuous on $(0, n)$.

The Hausdorff dimension $\operatorname{dim} W(F, \psi ; m, n)$ of the set

$W(F, \psi ; m, n)=\left\{X \in \mathbb{R}^{m n}: F(\langle\boldsymbol{q} X\rangle)<\psi(\boldsymbol{q})\right.$ for infinitely many $\left.\boldsymbol{q} \in \mathbb{Z}^{m}\right\}$

will be estimated in terms of the lower order $\lambda_{1}$ of $1 / \psi_{1}$ and the lower order $\omega(s)$ of the $s$-volume of a cover for the star body

$$
S_{r}=F^{-1}([0, r)) \cap I^{n}=\left\{\boldsymbol{x} \in I^{n}: F(\boldsymbol{x})<r\right\},
$$


where $r>0$ and $I=\left[-\frac{1}{2}, \frac{1}{2}\right]$. It will then be determined in terms of the dimension of $W\left(F, \psi_{1} ; 1, n\right)$. This extends the simultaneous Diophantine approximation results of [5] to systems of linear forms and generalizes the results of [4] and [13]. It will also be used to determine the Hausdorff dimension of the set of real $m \times n$ matrices $X$ for which the inequality

$$
\prod_{j=1}^{n}\left|\left\langle\sum_{i=1}^{m} q_{i} x_{i j}\right\rangle\right|^{\alpha_{j} / A}<\prod_{i=1}^{m}\left(\bar{q}_{i}\right)^{-\tau}
$$

where $\alpha_{1}, \ldots, \alpha_{n}>0, A=\alpha_{1}+\ldots+\alpha_{n}$ and $\bar{q}=\max \{1,|q|\}$, holds for infinitely many $\boldsymbol{q} \in \mathbb{Z}^{m}$ (in [13], $\alpha_{j}=1$ for each $j=1, \ldots, n$ ).

Since

$$
\operatorname{dim}\left\{A_{1} \cup A_{2} \cup \ldots\right\}=\sup \left\{\operatorname{dim} A_{k}: k=1,2, \ldots\right\},
$$

in order to determine the Hausdorff dimension of $W(F, \psi ; m, n)$ it suffices to consider the dimension of the set

$$
\begin{aligned}
\widetilde{W}(F, \psi ; m, n) & =\left\{X \in I^{m n}: F(\langle\boldsymbol{q} X\rangle)<\psi(\boldsymbol{q}) \text { for infinitely many } \boldsymbol{q} \in \mathbb{Z}^{m}\right\} \\
& =W(F, \psi ; m, n) \cap I^{m n} .
\end{aligned}
$$

TheOREM 1. Let $F: \mathbb{R}^{n} \rightarrow \mathbb{R}$ be a distance function and suppose that for each $r>0$, the starbody $F^{-1}([0, r)) \cap I^{n}$ has a cover $\mathcal{C}_{r}$ of $n$-dimensional hypercubes $C$ with sides of length $\ell(C) \ll r$. Let $\omega(s)$ be the order of the s-volume $\ell^{s}\left(\mathcal{C}_{r}\right)$. Let $\psi: \mathbb{R}^{n} \backslash\{\mathbf{0}\} \rightarrow \mathbb{R}$ be an error function and suppose that the reciprocal $1 / \psi_{1}$ of $\psi_{1}: \mathbb{R} \backslash\{0\} \rightarrow \mathbb{R}^{+}$has lower order $\lambda_{1}$ satisfying

$$
\lim _{s \rightarrow 0+} \frac{\omega(s)}{n+1}<\frac{1}{\lambda_{1}}<\lim _{s \rightarrow n-} \omega(s) .
$$

Suppose further that for every $v>\omega\left(s_{0}\right)$,

$$
\sum_{\mid=q} \psi(\boldsymbol{q})^{v} \ll \psi_{1}(q)^{v} .
$$

Then

$$
\operatorname{dim} W(F, \psi ; m, n) \leq(m-1) n+s_{0}=m n+1-\lambda_{1} \omega\left(s_{0}\right),
$$

where $s_{0} \in(0, n)$ is the unique solution of

$$
\lambda_{1} \omega(s)=n-s+1 .
$$

Proof. Choose $t$ such that $(m-1) n+s_{0}<t<m n$ and put $s=$ $t-(m-1) n$ so that $s_{0}<s<n$. The number $\eta=\lambda_{1} \omega(s)-n+s-1>0$ since $\omega(s)$ increases with $s$ and $s>s_{0}$. Let $\varepsilon$ be an arbitrary number satisfying

$$
0<\varepsilon<\eta / 2\left(\lambda_{1}+\omega(s)\right) .
$$


The set $\widetilde{W}(F, \psi ; m, n)$ can be expressed in the 'lim-sup' form

$$
\widetilde{W}(F, \psi ; m, n)=\bigcap_{N=1}^{\infty} \bigcup_{q=N}^{\infty} \bigcup_{\mid} \bigcup B(\boldsymbol{p}, \boldsymbol{q} ; \psi(\boldsymbol{q})),
$$

where $\boldsymbol{p}$ runs through all integer vectors in $\boldsymbol{q}\left(-\frac{1}{2}, \frac{1}{2}\right]^{m n}$ (i.e., $\boldsymbol{p}=\boldsymbol{q} X$ for some $X$ in $\left.\left(-\frac{1}{2}, \frac{1}{2}\right]^{m n}\right)$ and for each $\boldsymbol{p} \in \mathbb{Z}^{n}, \boldsymbol{q} \in \mathbb{Z}^{m}$ and $r>0$,

$$
B(\boldsymbol{p}, \boldsymbol{q} ; r)=\left\{X \in I^{m n}: F(\boldsymbol{q} X-\boldsymbol{p})<r, \boldsymbol{q} X-\boldsymbol{p} \in\left(-\frac{1}{2}, \frac{1}{2}\right]^{n}\right\} .
$$

Now $B(\boldsymbol{p}, q ; r)=\left\{\boldsymbol{x} \in I^{n}: F(q \boldsymbol{x}-\boldsymbol{p})<r, q \boldsymbol{x}-\boldsymbol{p} \in\left(-\frac{1}{2}, \frac{1}{2}\right]^{n}\right\}$, where $q>0$, is a starbody centred at $\boldsymbol{p} / q$ and is contained in $S_{r} / q+\boldsymbol{p} / q$, since if $y \in q B(\boldsymbol{p}, q ; r)-\boldsymbol{p}$, then $F(\boldsymbol{y})=F(q \boldsymbol{x}-\boldsymbol{p})<r$. The cover $\mathcal{C}_{r}$ for $S_{r}$ gives a cover $\mathcal{B}(\boldsymbol{p}, q ; r)$ for $B(\boldsymbol{p}, q ; r)$ consisting of $n$-dimensional hypercubes $C$ with $\ell(C) \ll r / q$ and, by the definition of $\omega(s)$, with $s$-volume $\ell^{s}(\mathcal{B}(\boldsymbol{p}, q ; r))$ satisfying

$$
\ell^{s}(\mathcal{B}(\boldsymbol{p}, q ; r)) \ll \ell^{s}\left(\mathcal{C}_{r}\right) q^{-s} \ll r^{\omega(s)-\varepsilon} q^{-s}
$$

for any $\varepsilon>0$ and sufficiently small $r$. By Lemma 2 of [4] or by [13] there is a cover $\mathcal{H}(\boldsymbol{p}, \boldsymbol{q} ; r)$ say of $B(\boldsymbol{p}, \boldsymbol{q} ; r)$ by $m n$-dimensional hypercubes $H$ with $\ell(H) \ll r /|\boldsymbol{q}|$ and $t$-volume

$$
\ell^{t}(\mathcal{H}(\boldsymbol{p}, \boldsymbol{q} ; r)) \ll \ell^{s}(\mathcal{B}(\boldsymbol{p},|\boldsymbol{q}| ; r)) .
$$

Hence for each $N=1,2, \ldots, \widetilde{W}(F, \psi ; m, n)$ has a cover $\Lambda_{N}$ of hypercubes $C$ with $\ell(C) \ll 1 / N$ and with $t$-volume $\ell^{t}\left(\Lambda_{N}\right)$ satisfying

$$
\begin{aligned}
\ell^{s}\left(\Lambda_{N}\right) & \ll \sum_{q=N}^{\infty} \sum_{|=q|} \sum_{|\leq|} \ell^{t}(\mathcal{H}(\boldsymbol{p}, \boldsymbol{q} ; \psi(\boldsymbol{q}))) \\
& \ll \sum_{q=N}^{\infty} \sum_{|=q|} \sum_{|\leq|} \ell^{s}(\mathcal{B}(\boldsymbol{p},|\boldsymbol{q}| ; \psi(\boldsymbol{q}))) \\
& \ll \sum_{q=N}^{\infty} \sum_{|=q|} \sum_{|\leq|}|\boldsymbol{q}|^{-s} \psi(\boldsymbol{q})^{\omega(s)-\varepsilon}
\end{aligned}
$$

by (3) with $r=\psi(\boldsymbol{q})$ and $q=|\boldsymbol{q}|$. Hence for all $t>(m-1) n+s_{0}\left(\right.$ or $\left.s>s_{0}\right)$ and $N$ sufficiently large,

$$
\begin{aligned}
\ell^{t}\left(\Lambda_{N}\right) & \ll \sum_{q=N}^{\infty} q^{-s} \sum_{||=q} \psi(\boldsymbol{q})^{\omega(s)-\varepsilon} \sum_{|| \leq \mid} 1 \\
& \ll \sum_{q=N}^{\infty} q^{n-s} \sum_{||=q} \psi(\boldsymbol{q})^{\omega(s)-\varepsilon} \ll \sum_{q=N}^{\infty} q^{n-s} \psi_{1}(q)^{\omega(s)-\varepsilon}
\end{aligned}
$$

by (2). Hence by the definition of the lower order $\lambda_{1}$ of $1 / \psi_{1}$, for sufficiently 
large $N$,

$$
\begin{aligned}
\ell^{t}\left(\Lambda_{N}\right) & \ll \sum_{q=N}^{\infty} q^{n-s} q^{-\left(\lambda_{1}-\varepsilon\right)(\omega(s)-\varepsilon)} \\
& \ll \sum_{q=N}^{\infty} q^{n-s-\lambda_{1} \omega(s)-\varepsilon\left(\lambda_{1}+\omega(s)\right)} \ll \sum_{q=N}^{\infty} q^{-1-\eta / 2}
\end{aligned}
$$

by the choice of $\eta$ and $\varepsilon$. Thus $\ell^{t}\left(\Lambda_{N}\right) \rightarrow 0$ as $N \rightarrow \infty$ and it follows that $\operatorname{dim} W(F, \psi ; m, n) \leq(m-1) n+s_{0}$.

By using the above result and the following general lower bound, the Hausdorff dimension of $W(F, \psi ; m, n)$ can be expressed in terms of the dimension of $W\left(F, \psi_{1} ; 1, n\right)$. To do this a slight additional restriction on the order $\omega$ is needed. It suffices to assume that $\omega(s)$ is strictly increasing, or that in the cover $\mathcal{C}_{r}, \ell(C) \gg r$, or that since $\omega(s)$ is a convex function of $s$, for some $\delta>0, \omega(s)$ is not constant for $s>s_{0}-\delta$ or that $\ell(C)^{s} \ll r^{\omega(s)}$. In the applications made, each of these conditions is fulfilled.

LEMma 1. Let $F: \mathbb{R}^{n} \rightarrow \mathbb{R}$ be a distance function and let $\psi: \mathbb{R}^{n} \backslash\{\mathbf{0}\} \rightarrow \mathbb{R}$ be any function. Then

$$
\operatorname{dim} W(F, \psi ; m, n) \geq(m-1) n+\operatorname{dim} W\left(F, \psi_{1} ; 1, n\right) .
$$

Proof. [4], Lemma 1.

TheOREM 2. Let $F: \mathbb{R}^{n} \rightarrow \mathbb{R}$ be a distance function and suppose that for each $r>0$, the starbody $F^{-1}([0,1)) \cap I^{n}$ has a cover $\mathcal{C}_{r}$ of $n$-dimensional hypercubes $C$ with sides of length $\ell(C) \ll r$. Let $\omega(s)$ be the lower order of the $s$-volume $\ell^{s}\left(\mathcal{C}_{r}\right)$ and suppose that $\omega(s)$ is strictly increasing. Let $\psi: \mathbb{R}^{n} \backslash$ $\{\mathbf{0}\} \rightarrow \mathbb{R}$ be an error function and suppose that for $s>\operatorname{dim} W\left(F, \psi_{1} ; 1, n\right)$,

$$
\sum_{\mid=q} \psi(q)^{\omega(s)} \ll \psi_{1}(q)^{\omega(s)}
$$

and

$$
\sum_{q=1}^{\infty} q^{n-s} \psi_{1}(q)^{\omega(s)}<\infty
$$

Then

$$
\operatorname{dim} W(F, \psi ; m, n)=(m-1) n+\operatorname{dim} W\left(F, \psi_{1} ; 1, n\right) .
$$

Proof. The proof is similar to that of Theorem 1 except that the lower bound of the lemma coincides with the upper bound. Write $h=$ $\operatorname{dim} W(F, \psi ; m, n)$ and assume without loss of generality that $h<n$. Choose $t$ such that $(m-1) n+h<t<m n$ and put $s=t-(m-1) n$ so that $h<s<n$. Since $\omega(s)$ increases strictly with $s, \omega(s)-\omega(h)>0$. Let $\varepsilon$ be an arbitrary number satisfying $0<\varepsilon<\omega(s)-\omega(h)$. 
As in Theorem 1, for each $N=1,2, \ldots, \widetilde{W}(F, \psi ; m, n)$ has a cover $\Lambda_{N}$ consisting of hypercubes $C$ with $\ell(C) \ll 1 / N$ and with $t$-volume satisfying

$$
\ell^{t}\left(\Lambda_{N}\right) \ll \sum_{q=N}^{\infty} \sum_{|=q|} \sum_{|\leq|}|\boldsymbol{q}|^{-s} \psi(\boldsymbol{q})^{\omega(s)-\varepsilon} .
$$

Hence for all $t>(m-1) n+h$ (i.e., $s>h)$ and $N$ sufficiently large,

$$
\ell^{t}\left(\Lambda_{N}\right) \ll \sum_{q=N}^{\infty} q^{n-s} \psi_{1}(q)^{\omega(s)-\varepsilon} \ll \sum_{q=N}^{\infty} q^{n-s^{\prime}} \psi_{1}(q)^{\omega\left(s^{\prime}\right)},
$$

where $\omega\left(s^{\prime}\right)=\omega(s)-\varepsilon$, so that by the choice of $\varepsilon$ and since $\omega$ is strictly increasing, $h<s^{\prime}<s$. Thus $\ell^{t}\left(\Lambda_{N}\right) \rightarrow 0$ as $N \rightarrow \infty$ by (5) and it follows that $\operatorname{dim} W(F, \psi ; m, n) \leq(m-1) n+h$.

The complementary inequality $\operatorname{dim} W(F, \psi ; m, n) \geq(m-1) n+h$, and hence the theorem, follow from the lemma.

From now on take $\psi_{1}(x)=x^{-\tau}$ for $x, \tau>0$, so that $\lambda_{1}=\tau$, and write

$$
W\left(F, \psi_{1} ; 1, n\right)=W_{F}(\tau ; n) .
$$

It is shown in [5] that when $\omega(s) \tau>1, \operatorname{dim} W_{F}(\tau ; n) \leq s_{0}$, where $\tau \omega\left(s_{0}\right)=$ $n-s_{0}+1$. When $F(\boldsymbol{x})=|\boldsymbol{x}|$ or more generally when $F$ is a gauge function (i.e., when $F^{-1}(0)=\{\mathbf{0}\}$, see [8], p. 8), then $\omega(s)=s$ and $\operatorname{dim} W_{F}(\tau ; n)=$ $(n+1) /(\tau+1)$ for $\tau>1 / n$. Since $(n+1) /(\tau+1)$ is a continuous decreasing function of $\tau$ which equals $n$ when $\tau=1 / n$, since $\operatorname{dim} W_{F}(\tau ; n) \leq n$ and since $\tau \geq \tau^{\prime}$ implies that $W_{F}(\tau ; n) \subseteq W_{F}\left(\tau^{\prime} ; n\right)$, it follows that

$$
\operatorname{dim} W_{F}(\tau ; n)=(n+1) /(\tau+1)
$$

holds for $\tau \geq 1 / n$. For if $\operatorname{dim} W_{F}(1 / n ; n)=\theta<n$, then for $\tau-1 / n>0$ and sufficiently small, $W_{F}(\tau ; n)>\theta$, a contradiction. The estimate (4) is essential for Theorem 2 and does not hold for the error function $\psi(\boldsymbol{x})=$ $|\boldsymbol{x}|^{-\tau}$ when $m \geq 2$; indeed, in this case the Hausdorff dimension is larger and $\operatorname{dim} W(F, \psi ; m, n)=(m-1) n+(m+n) /(\tau+1)$ when $\tau \geq m / n[2]$.

COROLlary 1. Suppose that $F: \mathbb{R}^{n} \rightarrow \mathbb{R}$ is a gauge function and that $\psi: \mathbb{R}^{n} \backslash\{\mathbf{0}\} \rightarrow \mathbb{R}$ satisfies $\psi_{1}(x)=\psi(x, 0, \ldots, 0)=x^{-\tau}$ and

$$
\sum_{||=q} \psi(\boldsymbol{q})^{s} \ll q^{-\tau s}
$$

for each $s>(n+1)(\tau+1)$. Then for $\tau \geq 1 / n$,

$$
\operatorname{dim} W(F, \psi ; m, n)=(m-1) n+\frac{n+1}{\tau+1} .
$$

Proof. By [5], $\omega(s)=s$ and by [6] or [10] and by the above remarks on the continuity of the dimension, $\operatorname{dim} W_{F}(\tau ; n)=(n+1) /(\tau+1)$ when 
$\tau \geq 1 / n$. The estimate (5) holds since when $s>(n+1) /(\tau+1)$,

$$
\sum_{q=1}^{\infty} q^{n-s} q^{-\tau s}<\infty
$$

and the corollary follows.

This result was obtained in [4] but the condition corresponding to (5) was omitted; this does not affect the applications made there. Note that since $\tau s_{0}=n-s_{0}+1$ when $s_{0}=(n+1)(\tau+1)$, it follows from Theorem 1 and the above remarks on the continuity of the dimension that $\operatorname{dim} W(F, \psi ; m, n) \leq$ $(m-1) n+(n+1) /(\tau+1)$ when $\tau \geq 1 / n$.

The function $F$ given by $F(\boldsymbol{x})=\prod_{j=1}^{n}\left|x_{j}\right|^{1 / n}$ is evidently a distance function; $F$ is also of interest in the geometry of numbers ([8], Chapter 4$)$ and Diophantine approximation ([12], p. 69). In [13], Yu extended [1] to systems of linear forms and showed (using different notation) that when $\psi(\boldsymbol{x})=$ $\prod_{i=1}^{m}\left(\bar{x}_{i}\right)^{-\tau}$, where $\tau>1 / n$ and where for each real $x, \bar{x}=\max \{|x|, 1\}$, then

$$
\operatorname{dim} W(F, \psi ; m, n)=m n-1+\frac{2}{\tau n+1} .
$$

Using Theorem 2, we now extend Yu's result.

COROllaRY 2. Let $\alpha_{1}, \ldots, \alpha_{n}$ be positive numbers with $\alpha=\max \left\{\alpha_{j}\right.$ : $1 \leq j \leq n\}$ and $A=\alpha_{1}+\ldots+\alpha_{n}$. Let $\Phi: \mathbb{R}^{n} \rightarrow \mathbb{R}$ be given by

$$
\Phi(\boldsymbol{x})^{A}=\prod_{j=1}^{n}\left|x_{j}\right|^{\alpha_{j}}
$$

and $\psi: \mathbb{R}^{n} \rightarrow \mathbb{R}$ by

$$
\psi(\boldsymbol{x})=\prod_{i=1}^{m}\left(\bar{x}_{i}\right)^{-\tau} .
$$

Then if $\tau \geq \alpha / A$, the set

$$
W_{\Phi}(\tau ; m, n)=\left\{X \in \mathbb{R}^{m n}: \Phi(\langle\boldsymbol{q} X\rangle)<\psi(\boldsymbol{q}) \text { for infinitely many } \boldsymbol{q} \in \mathbb{Z}^{m}\right\}
$$

has Hausdorff dimension

$$
\operatorname{dim} W_{\Phi}(\tau ; m, n)=m n-1+\frac{2 \alpha}{\tau A+\alpha} .
$$

Proof. Evidently $\Phi$ is a distance function and the restriction $\psi_{1}$ of $\psi$ is given by $\psi_{1}(q)=q^{-\tau}, q \in \mathbb{N}$. Now $\omega(s)=A /(s-n+1) / \alpha$ for $n-1<s<n$ ([5], Lemma 2$)$ and is strictly increasing and $\operatorname{dim} W_{\Phi}(\tau ; 1, n)=$ $n-1+2 \alpha /(\tau A+\alpha)$ when $\tau>\alpha / A$ ([5], Theorem 2). By continuity this 
formula also holds when $\tau=\alpha / A$. The sum corresponding to (5) is

$$
\sum_{q=1}^{\infty} q^{n-s} q^{-\tau A(s-n+1) / \alpha}
$$

which is finite since $\tau A(s-n+1) / \alpha>n-s+1$ when $s>n-1+2 \alpha /(\tau A+\alpha)$. Finally, it has to be shown that the inequality

$$
\sum_{\mid=q} \prod_{i=1}^{m}\left|\bar{q}_{i}\right|^{-\tau A(s-n+1) / \alpha} \ll q^{-\tau A(s-n+1) / \alpha}
$$

corresponding to (4) holds when $s>n-1+2 \alpha /(\tau A+\alpha)$. Now for each $q \in \mathbb{N}$

$$
\begin{aligned}
\sum_{\mid=q} \prod_{i=1}^{m}\left|\bar{q}_{i}\right|^{-\tau A(s-n+1) / \alpha} & \ll q^{-\tau A(s-n+1) / \alpha} \sum_{i=1}^{m} \prod_{j \neq i} \sum_{q_{j}=-q}^{q}\left|\bar{q}_{j}\right|^{-\tau A(s-n+1) / \alpha} \\
& \ll q^{-\tau A(s-n+1) / \alpha}\left(\sum_{k=1}^{\infty} k^{-\tau A(s-n+1) / \alpha}\right)^{m-1} .
\end{aligned}
$$

But $s>n-1+2 \alpha /(\tau A+\alpha)$ implies that $\tau A(s-n+1) / \alpha>2 \tau A /(\tau A+\alpha) \geq 1$ when $\tau \geq A / \alpha$ and so (6) holds. Hence by Theorem 2 and continuity, when $\tau \geq A / \alpha$,

$$
\operatorname{dim} W_{\Phi}(\tau ; m, n)=(m-1) n+n-1+\frac{2 \alpha}{\tau A+\alpha}=m n-1+\frac{2 \alpha}{\tau A+\alpha} .
$$

Acknowledgement. We would like to thank the referee for pointing out some obscurities.

\section{References}

[1] J. D. Bovey and M. M. Dodson, The fractional dimension of sets whose simultaneous rational approximations have errors with a small product, Bull. London Math. Soc. 10 (1978), 213-218.

[2] - - - The Hausdorff dimension of systems of linear forms, Acta Arith. 45 (1986), 337-358.

[3] J. W. S. Cassels, An Introduction to the Geometry of Numbers, Grundlehren Math. Wiss. 99, Springer, Berlin 1959.

[4] M. M. Dodson, A note on the Hausdorff-Besicovitch dimension of systems of linear forms, Acta Arith. 44 (1985), 87-98.

[5] —, Star bodies and Diophantine approximation, J. London Math. Soc. 44 (1991), $1-8$.

[6] H. G. Eggleston, Sets of fractional dimensions which occur in some problems of number theory, Proc. London Math. Soc. 54 (1951-1952), 42-93.

[7] K. J. Falconer, The Geometry of Fractal Sets, Cambridge Tracts in Math. 85, Cambridge University Press, Cambridge 1985. 
[8] P. Gruber and C. G. Lekkerkerker, Geometry of Numbers, North-Holland, Amsterdam 1987.

[9] W. K. Hayman, Meromorphic Functions, Oxford Math. Monographs, Clarendon Press, Oxford 1964.

[10] V. Jarník, Über die simultanen diophantischen Approximationen, Math. Z. 33 (1931), 505-543.

[11] H. Rüssmann, On the one-dimensional Schrödinger equation with a quasi-periodic potential, Ann. New York Acad. Sci. 357 (1980), 90-107.

[12] V. G. Sprindžuk, Metric Theory of Diophantine Approximations, translated by R. A. Silverman, V. H. Winston \& Sons, Washington, D.C., 1979.

[13] K. Yu, Hausdorff dimension and simultaneous rational approximation, J. London Math. Soc. 24 (1981), 79-84.

DEPARTMENT OF MATHEMATICS

UNIVERSITY OF YORK

YORK YO1 5DD, ENGLAND

Received on 24.4.1990

and in revised form on 26.7.1991 